\title{
Priority control of berth allocation problem in container terminals
}

\author{
Evrim Ursavas ${ }^{1}$
}

Published online: 7 September 2015

(c) The Author(s) 2015. This article is published with open access at Springerlink.com

\begin{abstract}
This paper presents a decision support system for the core problem of berth allocation decision in a container terminal. The allocation of berths to the calling vessels is complex with the fact that different service level requirements are required for different vessels. Terminal managers demand for effective decision support systems that would aid them with the allocation problem considering service priorities. Consequently, this study provides a DSS, built by a dynamic discrete-event simulation model embedded with an optimization tool that determines the priority controls for the berth allocation to the calling vessels. To show the practical application of the DSS, a comprehensive case study from a Turkish container terminal considering the current state and future expansion plans that also provides an indication of the usability aspect of the program on other ports around the world has been conducted. Further experiments are conducted based on data from the Port of Rotterdam. The DSS presented in this study may help port authorities in determining more efficient allocation decisions within a container terminal.
\end{abstract}

Keywords Port management · Simulation optimization · Berth allocation · Container terminal operations

\section{Introduction}

With the high demand of sea trade, container transportation has been the core of modern logistics, and container terminals are considered as key nodes of international transportation. Container terminals are complex dynamic systems intertwined with many activities. Among those operations berth allocation problem can be considered as one of the major issues as it is this operation that drives the whole port management process. When a vessel arrives at a port, it must be anchored at a specified berth by the terminal planners. In Multi-User Terminals

Evrim Ursavas

e.ursavas@rug.nl

1 Department of Operations, Faculty of Economics and Business, University of Groningen, Groningen, The Netherlands 
(MUT) where berths are dynamically allocated to calling vessels and are not always assigned to specific berth locations such as in Dedicated Terminals (DT), berth planning becomes the root of all operations. An inefficient decision in the berth allocation phase affects all the other applications connected to this and may increase the service period and costs. Existing literature has demonstrated that employing flexible vessel-berth-order allocation without taking into account the First-Come-First-Served (FCFS) rule, higher productivity can be obtained (Imai et al. 2008). Following the FCFS rule and treating every vessel without any differentiated priority may have severe results up to the loss of current leading customers. Terminal managers are therefore enforced to operate their berths under intelligent strategies. In practice, vessels with high container volume prefer to be given priority over small vessels. In general the ship priority depends on the total throughput per shipping line. Accordingly, the ship size can be regarded as a variable for the priority index which is often closely correlated to the significance of the shipping company (Imai et al. 2003). However, terminal operators must also consider the issue of smaller vessels where they may be dominated by large vessels having much higher handling times. From the above discussions, it is apparent that a decision support tool that would help terminal operators in making efficient berth allocation decisions under dynamic terminal settings is of high necessity.

The contribution of this article consists of providing terminal managers a decision support tool, convenient for use in different type of berth layouts, for the berth allocation problem considering service level differentiations amongst vessels. The DSS employs an architecture to generate solutions for the user intervened problem cases. Given the importance of stochastic nature of the container terminal operations, a discrete event simulation model, with an embedded optimization module based on meta-heuristics, is developed for the core component of the decision support system.

The remainder of this paper is organized as follows: Following section will put forward the related literature. The problem will be explained in detail in Sect. 3. Next, proposed system structure will be presented. Subsequently, system applications and analysis will be presented. Finally, the last section will be devoted to concluding remarks and future work.

\section{Literature review}

The need for effective decision making strategies for managing container terminal operations has become certainly obvious and therefore the area has attracted many researchers into the subject (Carlo et al. 2013; Vis and Koster 2003; Steenken et al. 2004; Stahlbock and Voß 2008). In this review, studies handling berth allocation problem considering vessel priorities will be summarized first. Next, studies that capture the stochastic nature of the problem will be put forward. Last, previous literature on decision support systems developed for use in container terminals will be presented.

With regard to the studies dealing with the berth allocation problem and together considering different levels of vessel priorities, Imai et al. (2003) employed a genetic algorithm based heuristic to solve the nonlinear problem by defining priorities through assigning weights to the vessels related by their handling volumes within the objective function. Hansen et al. (2008) imposed ship dependent premiums and penalty costs for handling priority issues and aimed to minimize total costs made up of waiting, handling, earliness and lateness costs. In Imai et al. (2007), a bi-objective berth allocation problem where weights are employed to distinguish between the dissimilar vessel priorities minimizing vessel delays and total service time is presented. Saharidis et al. (2010) categorized the vessels as preferential or 
non-preferential and have considered the maximization of preferential customer satisfaction to be more important than port's total throughput. They used hierarchical optimization framework, using hierarchical structure that distinguishes between two contradictory objectives port managers come across for the berth allocation problem. Guan et al. (2002) assigned weights to the vessels according to their sizes which were used within the objective function where the aim was to minimize the total weighted completion time of vessel service. Guan and Cheung (2004) defined a weight coefficient for each vessel and they optimized the total weighted flow time using a method that combines tree procedure and a heuristic. Study by Cordeau et al. (2005) is based on multi depot vehicle routing problem with time windows in which the objective is the minimization of the total weighted service time. Boile et al. (2006) linearized the berth allocation formulation presented by Imai et al. (2003) and proposed heuristic solutions to solve large instances of the problem with the objective to minimize the weighted total service time. Golias et al. (2006) introduced time window service deadlines with the aim to minimize late vessel departures and maximize early/timely departures of vessels using two separate objective functions. Subsequently, Boile et al. (2007) presented a multiobjective optimization approach where customers were differentiated by the use of a different objective function for each customer. Lai and Shih (1992) proposed a heuristic algorithm for berth allocation problem considering a FCFS policy for more efficient terminal usage in the HIT terminal of Hong Kong. Imai et al. (2008) studied BAP at a terminal where the berth capacities are very limited. They considered berth allocation at the restricted space, which gives the possibility of allocating some ships to another terminal. This study contributes to the literature on berth allocation by extending the work on priority considerations through a decision tool for determining priority controls based on simulation optimization. The priority controls are studied in further detail by considering additional factors such as berth-vessel link and different terminal configurations.

One crucial aspect of container terminal operations is the presence of uncertainty. The positioning problem of the vessels in the decision space without overlapping each other and while satisfying several constraints is in the class of NP. In such problems, it may not be possible to find short-time optimal solutions for the problems arising from the complicated practice situations. Instead, the objective becomes to find satisfactory results within reasonable time. This need has led to a significant stimulation in the development of heuristic solution methods. Indeed, heuristics and simulation are the methods widely used for the solution of the berth allocation problem. Studies reflecting the presence of uncertainty whilst planning the container terminal operations are relatively few. For the berth allocation problem, Zhou and Kang (2008) have used the genetic algorithm to search through the solution space and compared it with the greedy algorithm. The procedure of the algorithm is designed so as to reduce the search space based on the characteristics of the optimal solution. Constraints of the stochastic model are considered in the processes of individuals coding, initializing, crossover and mutation operations and also within the fitness function by employing sufficient punishment value. The genetic algorithm proposed has significantly improved the greedy algorithm solutions. Golias et al. (2007) proposed a conceptual formulation and four solution approaches for the berth allocation problem: Markov Chain Monte Carlo Based Heuristic, Stochastic Online Scheduling Based Heuristic, Deterministic Based Heuristic and Genetic Algorithm Based Approach. Han et al. (2010) worked on the problem with stochastic arrival and handling times. A simulation based genetic algorithm search procedure is applied to generate schedules proactively. The procedure applies a genetic algorithm framework, and incorporates a simulation module using Monte Carlo sampling to evaluate the performance of each single chromosome. The search procedure terminates when the maximum number of generations is achieved. Several researchers have applied the simulated annealing heuristic 
for solving the quayside related problems (Zhen et al. 2011; Du et al. 2010; Rajeeva and Chung 2006). The Simulated Annealing is based on cooling behavior of heated solid metal (Kirpatrick et al. 1983). Discrete-event simulation is also utilized to tackle the problem under stochastic nature (Yun and Choi 1999; Kim et al. 2003; Parola and Sciomachen 2005; Vis 2006; Pietro et al. 2008). Vis (2006) compared the performance of manned straddle carriers and automated stacking cranes in container terminals by means of a simulation study. The data used in the study by Pietro et al. (2008) is based on Gioia Tauro Container Terminal in Italy. First, a queuing network model is proposed and described for the berth allocation problem. Due to the complexity of the problem and the need for dynamic evaluation of decision policies using an analytical approach to the solution the model is discouraged and discrete-event simulation model is performed.

Subsequently, decision support systems that deal with the operations and planning of containers developed by researchers in the field will be presented. Bandeira et al. (2009) developed a decision support system that considers the flow of full containers together with the flow of empty containers. The case modelled as a multiple-depot vehicle scheduling problem is tackled in two interconnected stages where first allocation and movement of containers are determined and then this static solution is unfolded in a time schedule. Murty et al. (2005) proposed a decision support system where numerous interrelated daily decisions at a container terminal are handled. The aim of the DSS use is to minimize the berthing time of vessels, the resources needed for handling the workload, the waiting time of customer trucks, and the congestion on the roads and at the storage blocks and docks inside the terminal as well as to make the best use of the storage space. Ngai et al. (2007) presented the development of a prototype system in a container depot with radio frequency identification capabilities. Shen and Khoong (1995) proposed a decision support system using network optimization to model empty container repositioning.

This study aims to fill a gap in the literature by further incorporating real life aspects during actual implementation of the berth allocation problem. In summary, this study contributes to the literature on decision support systems with the following features:

- A novel dynamic priority control structure is embedded within the system which is designed for use in container terminal operations reflecting practical restrictions.

- Uncertainty in vessel arrivals and handling times are reflected to provide practically meaningful results.

- The DSS is convenient for use in different type of berth layouts such as peninsula type layouts, continuous quays or indented berth structure that is used for fast-handling of mega-container ships.

- Berths of different types working with individual productivity rates can be entered into the system.

- Definition of vessel classes can be based on different features such as vessel size and TEU (twenty-foot equivalent unit) volume.

These new features lead to a novel system for the berth allocation problem. The proposed model-driven decision support system is evaluated through an extensive case study in the container terminal in Izmir, Turkey and data from the Port of Rotterdam in the Netherlands. Real-world features to be included are put forward by the port decision makers and on site observations. High flexibility is attained by a good level of parameterization enabling the decision maker to use the DSS under numerous settings that may appear in practice. The DSS is then validated by the users and the practical usability is found satisfactory and suitable. The next section is dedicated to explaining the details of the studied problem. 


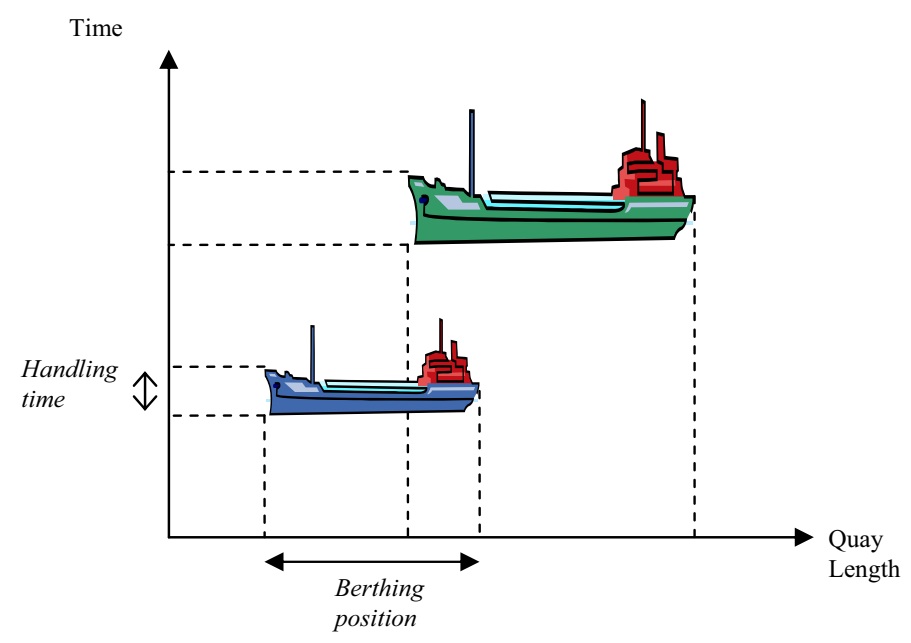

Fig. 1 Berth allocation problem

\section{Berth allocation problem}

Berth Allocation Problem in a container terminal can be described as the problem of allocating berth space to vessels. It aims to optimally assign vessels to berthing space along a quay. Figure 1 illustrates the problem in a two-dimensional space.

The strategy used when determining the berth-vessel link directly affects the port performance indicators. There are different strategies that may be followed; where the most common ones are first come first served, largest vessel first and shortest job first policies. Port authorities state that more sophisticated strategies require the use of efficient decision support tools and strategies that allow for differentiating service levels for different customer classes are considered as an essential support for effective use of berth facilities.

In world terminals, different types of terminal layouts in terms of berth characteristics may be observed. A major difference affecting the berth allocation strategy to be followed is the existence of dissimilarities among handling equipment, proximity to storage spaces and facilities between berths. Overall, these have an effect on the handling time of vessels serviced at specific berths. It may also be the case that berths in a container terminal has approximately similar handling rates. This is more common in terminal layouts where a peninsula shaped land space is used for berthing. Figure 2 depicts a layout of a terminal with a peninsula structure. This type of layout can often be observed in terminals around the world which gives the advantage of having longer quay space and similar proximity to the container storage yard which is typically placed in the centre. For instance, this layout can be seen in the ECT Container Terminal within the Port of Rotterdam in the Netherlands and at the Port of Izmir in Turkey.

As vessels of different types enter into the port they are directed to berths where there is available space. In case of congestion, they are held at the port horizon until they are directed to the quay queues following terminal planner instructions. In practice, the vessels are accompanied by tug boats and terminal personnel to the determined quay where they are soon to be berthed. Here, the terminal planner needs the support of a DSS to decide where to direct the vessels. Accordingly, the proposed DSS incorporates the following allocation strategies and priority control procedures for use in different terminal settings. 
Fig. 2 Peninsula shaped terminal layout

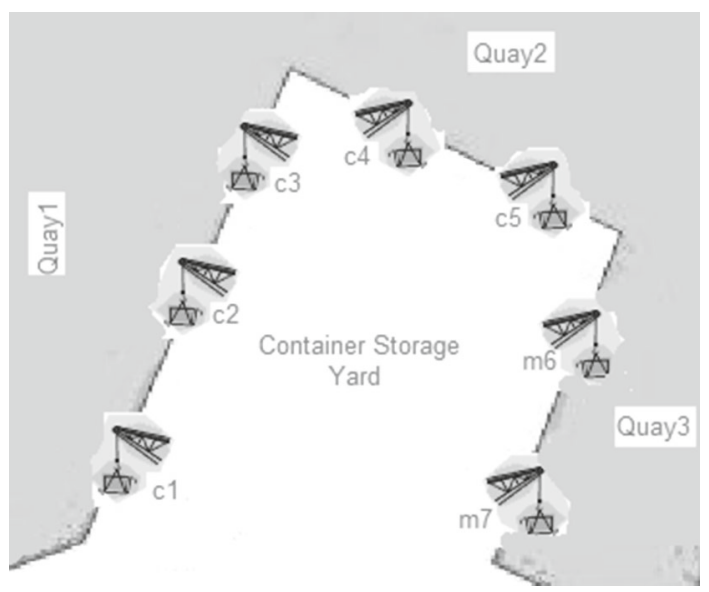

Procedure A Dedicated terminal configuration where specific berths are exclusively reserved for specific shipping companies are used. This strategy is straightforward to apply in practice, however it is still included within DSS features to allow for comparison of different polices for tactical decisions.

Procedure $B$ Vessels kept at the port horizon are directed to the first available berth from a single queue. Vessel arriving first will be served first.

Procedure $C$ Vessels with the shortest processing time waiting at the port horizon will be served first.

Procedure D In case of congestion, vessels are directed to the quay queues with the least number of waiting vessels. The waiting line of quays is organized by a priority control mechanism where the priority of each vessel is increased dynamically through time based increments with regard to its vessel class. Priority control is managed by the optimization module using scatter search methodology which determines priority increase index during the waiting period of each type of vessel class to maximize the total throughput of the system. Let us define $p r_{k}(\mathrm{t})$ as the instantaneous priority index for class $k$ vessel at time $t$. At time $t+\Delta t$ the priority index will be incremented by the amount computed through the system. Accordingly, vessels with longer waiting times will have priority over the vessels of the same class. However, it is possible for a vessel $j$ of class $k$ arriving at time $t+\Delta t$ to have higher priority over the previously arrived vessels within lower class.

Procedure E In case of berths with different handling rates, the handling rate of each berth class should also be considered when directing the vessels toward quays. Vessels are held at the port horizon until total number of vessels in quay queues of different handling rate levels meet certain levels computed by preference index $\pi_{j}$ of vessel $j$. Users have the option of assigning $\pi_{j}$ values through the user interface. Vessels are then directed towards the quay where they are soon to be serviced. The quay that the vessel will be assigned to is controlled by the priority control mechanism. Operators will keep track of the number of vessels waiting in the waiting line for each berth class. When a defined threshold level for the berth class is met, the vessel will be allowed to enter the quay. These threshold levels are dependent on the vessel and berth classes and are determined through the optimization module using scatter search methodology. Let us define $\tau_{k m}$ as the threshold level for vessel class $k$ and berth class $m$. Then, a vessel $j$ within class $k$ will enter the quay when the number of vessels waiting will meet $\tau_{k m}$. 
The first three policies A, B and C are more general and commonly used by container terminal planners. Policies D and E proposed by this study are more complex and require the additional support of decision support systems. The obtained critical levels through those polices has a critical impact on container terminals' performance indicators. Studies focusing on manufacturing companies' production capacity levels have also underlined the need for such strategies with different characteristics (Shi et al. 2014; Zhou et al. 2007, 2009). Figure 3 illustrates the overall system view of the mentioned polices where arrival rate for a class $-k$ vessel is denoted by $\lambda_{k}$ and service rate at class- $m$ berth is indicated by $\mu_{m}$.

Following section is devoted to the explanation of the developed system's architecture that embraces the aforementioned policies.

\section{System architecture}

The model-driven DSS is a software designed to run in a PC environment under Microsoft Windows. It employs an architecture to generate solutions for the user intervened problem cases. Major components of the DSS contain the user interface for input parameters, report visualization, the database management system for storing the data related with the terminal and the core of the DSS where the model is solved in compliance with the solution algorithm. Microsoft Access is used for the database. The procedures and the interaction with the simulation based optimization algorithm are developed by Visual Basic 6.0. Simulation is developed with Rockwell Software Arena 11.0. Figure 4 illustrates the main components of the model-driven DSS.

\subsection{Simulation optimization}

The solution methodology is built upon simulation and optimization modules that interact together exchanging model output results and decision variable values. Figure 5 depicts the procedure. The optimization module feeds the simulation module by supplying trial values for decision variable values. The solution value is computed through the execution of the simulation model. The generated output is returned to the optimization module to guide in the selection of a new trial solution. The process is repeated until a termination criterion is met. The solution with the best fitness value is returned to the user.

\subsection{Simulation model}

Figure 6 shows the simulation process view of events and activities. The system is initiated by the arrival announcements of vessels belonging to different classes. This is provided by the input data. To aid decision makers in analysing a situation the model is designed to provide a high degree of flexibility with a good level of parameterization. Primarily, the input of the DSS consists of parameters related with vessel classes, vessel size, container loads, arrival schedules, terminal physical characteristics, berth types and handling rates. Further parameters are related with preference indexes $\pi$ defined for vessels. Altering one or more of these parameters will have an impact on the whole system and consequently on the results reported to the user.

Vessel classifications may be based on vessel size or TEU volumes. Vessel size is often closely correlated by the significance of the company. Hence, port managers may opt for giving higher priority to more powerful companies. On the other hand, as mentioned by port authorities, TEU volumes handled within the port may also be used as an indication for the 


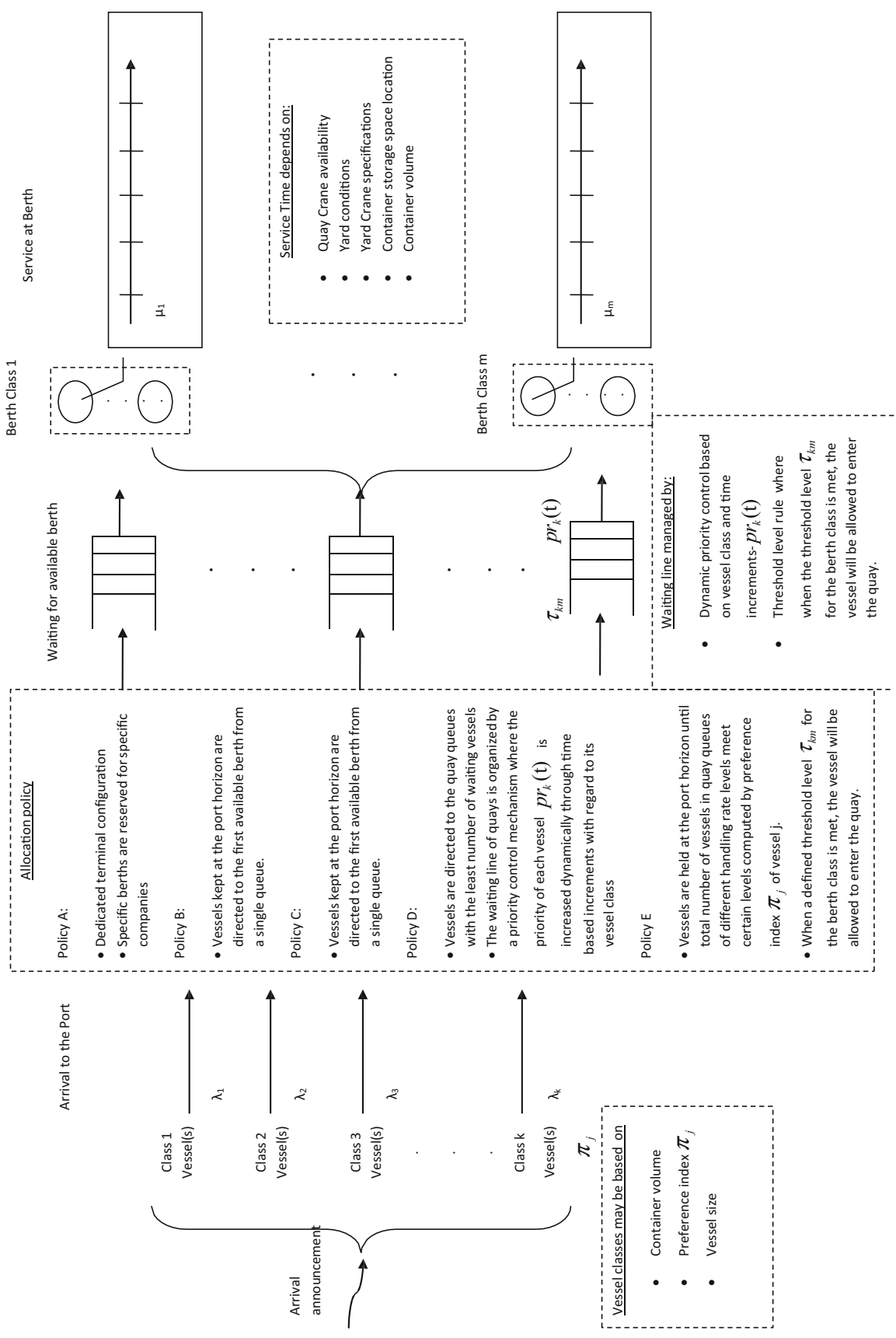

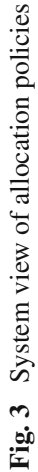




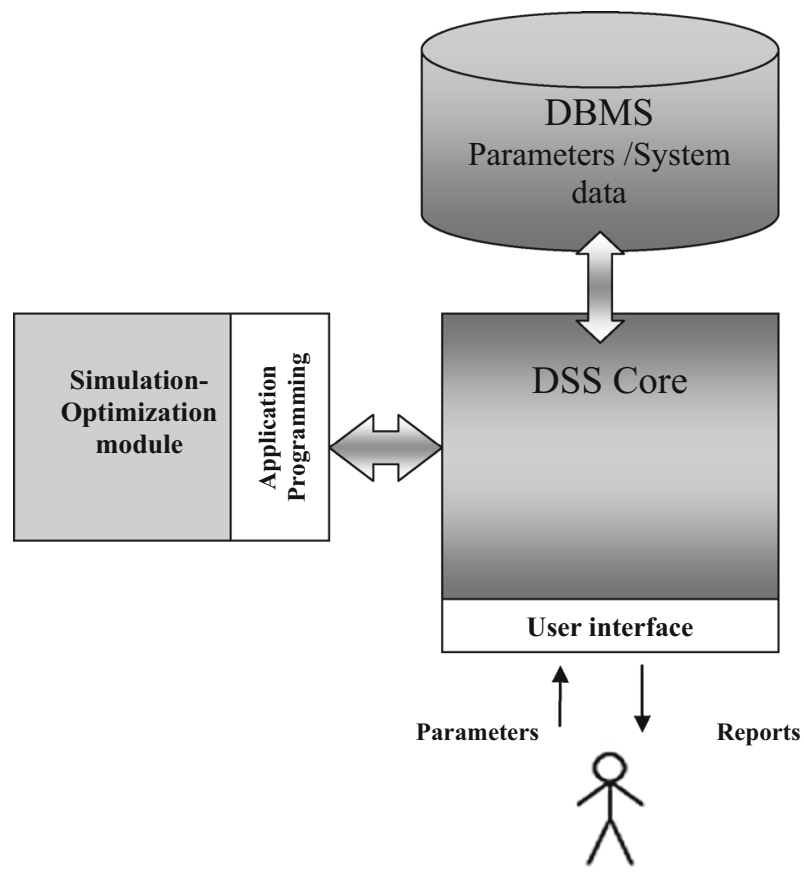

Fig. 4 DSS architecture

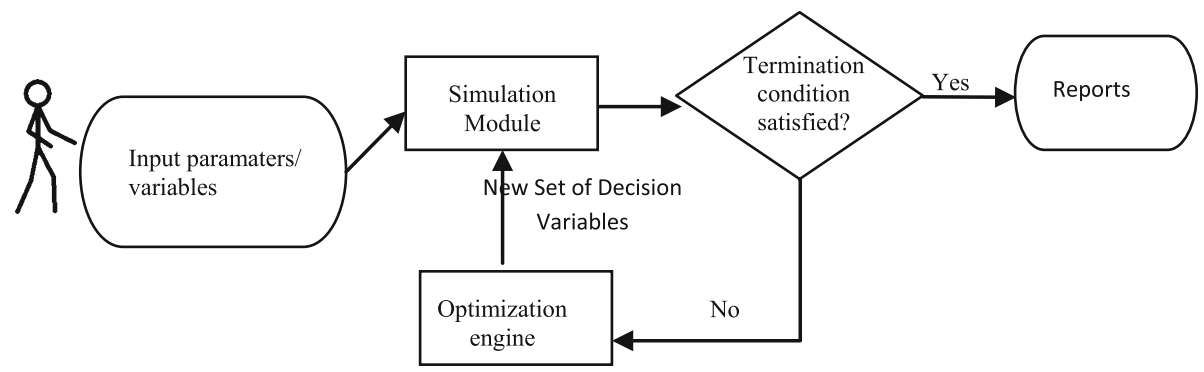

Fig. 5 Solution methodology

preference index. In this case, although less influential, terminal managers may still favour smaller shipping companies with high TEU volumes handled within the port. The vessel preferences based on the classification type used are managed by user input preferences and the model priority control mechanism. Users have the option of assigning preference weights to vessel classes through the user interface.

Following the arrival announcements, vessels are directed to their quays depending on the berth allocation policy. The berth allocation policy is determined through user options. The handling time of the vessel is dependent on the berth being served, container volumes of the vessels and yard conditions. Vessels completing the service can be unmoored from the berth and depart to the next port of destination. This ends the simulation for the entity. 


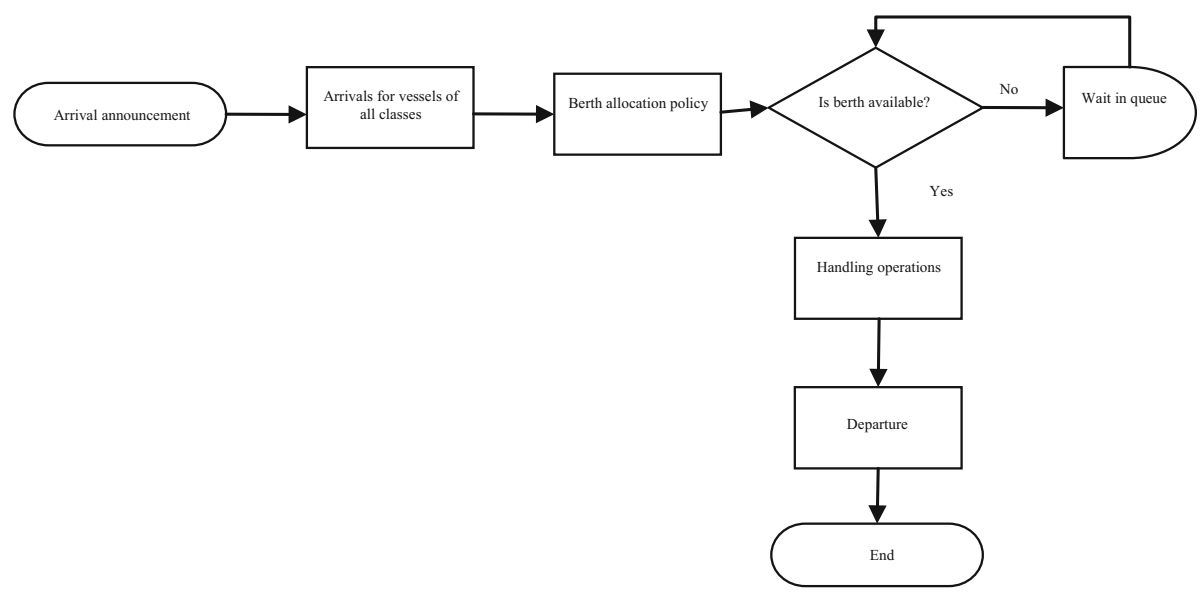

Fig. 6 Berth allocation process model

\subsubsection{Model validation}

In the context of computer models it is essential to check the accuracy of the model's representation of the real system with respect to its intended application (Sargent 2005). For the developed DSS, historical real data are used to obtain targets that are used as a measure to compare the model's outputs within $95 \%$ confidence intervals. Explicitly, weekly vessel arrival and departure data in 2011 at the Port of Izmir are examined. Table 1 shows the vessels with actual arrivals and departures for the week of November 7.

Current berth allocation strategy is generally based on dedicated terminal configuration (Procedure A) and allocation of berths according to the arrival sequence (Procedure B). More specifically, for particular shipping companies dedicated berths are utilized. These are carefully detected within the data for accurate evaluations. Average time between the arrival and the departure of a vessel is 19.96 hours distributed with gamma. Consequently, similar input setting is applied through the model to obtain an average service time through the model from 100 model replications. In $95 \%$ of repeated trials of the simulation model, the sample mean is reported as 20.39 within the interval \pm 1.73 half width. Accordingly, to assess the validity, the model estimate of the service time are compared to the past data of $\mu_{0}: 19.96$ hours. Comparison of the upper and lower control limits of the estimated data to the past data are 2.16 and 1.3, respectively. These differences are less than the established epsilon values, given as the maximum difference between the estimate and the real data to allow decisions to be based on simulation outputs.

\subsection{Optimization module:}

The priority control indexes and threshold levels within the system are managed by an optimization module in OptQuest. Microsoft Visual Basic 6.0 is used for the programming interfaces. The module uses the scatter search methodology to find the optimum set of values for the decision variables by executing the simulation model through running several scenarios for each set of inputs in order to maximize the objective function. The scatter search metaheuristic is similar to Genetic Algorithms in the sense that they both operate on a set of solutions that is maintained from iteration to iteration. The algorithm seeks to find an optimal 
Table 1 Vessel arrival and departure data of Port of Izmir

\begin{tabular}{llll}
\hline Ship id & Ship name & Arrival time & Departure time \\
\hline 1 & MSC1 & November 7th, 06:15 & November 7th, 23:15 \\
2 & NPT & November 7th, 17:00 & November 9th, 00:30 \\
3 & MRS1 & November 8th, 00:30 & November 8th, 16:15 \\
4 & HMS1 & November 8th, 00:00 & November 8th, 15:15 \\
5 & WW1 & November 8th, 01:50 & November 8th, 11:55 \\
6 & KPE1 & November 8th, 02:25 & November 9th, 00:15 \\
7 & VDB1 & November 8th, 08:50 & November 8th, 21:00 \\
8 & ORK1 & November 8th, 17:30 & November 9th, 22:15 \\
9 & WND1 & November 8th, 18:30 & November 9th, 15:10 \\
10 & LYQ1 & November 8th, 21:50 & November 9th, 08:20 \\
11 & MAR1 & November 9th, 02:00 & November 9th, 22:45 \\
12 & MSC2 & November 9th, 03:15 & November 9th, 17:00 \\
13 & MRS2 & November 9th, 16:30 & November 10th, 15:40 \\
14 & HMS2 & November 10th, 00:40 & November 10th, 13:30 \\
15 & WW2 & November 10th, 18:45 & November 12th, 06:00 \\
16 & KPE2 & November 11th, 05:30 & November 12th, 05:40 \\
17 & VDB2 & November 11th, 10:20 & November 12th, 16:45 \\
18 & ORK2 & November 11th, 15:35 & November 12th, 17:00 \\
19 & WND2 & November 12th, 09:45 & November 13th, 02:30 \\
20 & LYQ2 & November 12th, 10:15 & November 12th, 17:25 \\
21 & MAR2 & November 13th, 07:15 & November 14th, 10:30 \\
\hline & & &
\end{tabular}

solution to a problem defined on a set $X$ of bounded variables. Algorithm starts by generating an initial population of reference points that is a population of $X$ vectors. This includes the following points

$$
x_{i}=l_{i}+\left(u_{i}-l_{i}\right) / 2 \quad \text { for } i=1, \ldots, n
$$

where $L=\left(l_{i}: i=1, \ldots, n\right)$ is the set of lower bound values and $U=\left(u_{i}: i=1, \ldots, n\right)$ is the set of upper bound values for all $x_{i} \in X$. As in the genetic algorithms preserving a diverse set of solutions within the population is crucial for preventing sub-optimal solutions. The tolerance value to determine whether the two solutions are equal is set as 0.0001 . The population size is set to 100 . The fitness value of each solution is evaluated through the execution of the simulation model. Next, two reference points are selected from the population to form four offspring as follows:

$$
\begin{aligned}
& X_{3}=X_{1}+d \\
& X_{4}=X_{1}-d \\
& X_{5}=X_{2}+d \\
& X_{6}=X_{2}-d
\end{aligned}
$$

where

$$
d=\left(X_{1}-X_{2}\right) / 3
$$


Iteration ends by replacing the worst parent with the best offspring. More detailed description of the scatter search methodology can be seen in Glover et al. (1996).

Within the DSS, these control variables, denoted as $x_{i}$, are the priority change levels, that is the difference between $p r_{k}(\mathrm{t})$ and $p r_{k}(t+\Delta t)$ during the waiting period of each type of vessel class for Procedure D and threshold levels, $\tau_{k m}$, for vessel-berth class pairs in Procedure E. The algorithm searches for best possible values for these variables trying to maximize the throughput of the system. The objective function calculation for each candidate solution is calculated through the execution of the simulation model considering to minimize the expected total service time of vessels with a number of replications for a reliable estimation. The finishing time of vessels will be dependent upon the handling time of the vessels considering the berth class they are served at. This handling time is based on several factors such as crane availabilities, yard conditions and storage locations of containers. For Policy $\mathrm{D}$, the waiting line of berths, represented in the simulation component, is controlled by a priority control mechanism where the priority of each vessel is dynamically updated. The optimization module using scatter search methodology will determine those priorities $p r_{k}(\mathrm{t})$ defined on a set of bounded variables considering the best solution obtained within the set. For Policy E, vessels will be allowed to enter the quay when a defined threshold level $\tau_{k m}$ for the berth class is met. These threshold levels are dependent on the vessel and berth classes. As depicted in Fig. 5, the simulation model will be rerun with a new set of variables until a termination condition is met. More specifically the simulation will be executed on a set of bounded variables for the threshold levels and the optimization engine will search for the best possible set of values considering the overall objective.

\section{System applications and analysis}

The use of the DSS will be demonstrated through the application of the model in different type of container terminal settings. The terminal investigated thoroughly for the DSS demonstration is the container terminal in the Port of Izmir, Turkey, which actually enables the model's behaviour to be evaluated under different settings due to the future expansion plans that are also considered throughout the experiments. Hence, studying the port of Izmir will enable the model's usage to be assessed for more general use in other ports in the world. Mainly, the current state of the port and the future state where expansion of the port and the berthing facilities are planned are evaluated. For these two main scenarios, the model's behavior will further be assessed under different traffic volumes that will be based on vessel arrival data of the Uniport Container Terminal within the Port of Rotterdam. This provides the ability of demonstrating the model's usage under different arrival volumes that could be observed in larger ports around the world.

\subsection{Current terminal configuration experiments}

Currently, the container terminal in the Port of Izmir is divided into several quays, which in turn are divided into berths. Quays are 600, 400 and $360 \mathrm{~m}$ long. Each quay corresponds to a linear stretch of space in the terminal. The vessels can be berthed along the quay provided that the vessel length restrictions are met and there is space available after the mooring of other vessels. The container terminal lies in a peninsula shaped land where the store yard is positioned in the centre. As stated by the port authorities, the berths are considered to have similar handling rates. Yard equipment is similarly distributed among berths and the handling time depends on the container loads of the vessels and vessel restrictions. 
For this demonstration the vessels are classified according to their sizes. The three classes identified are as small $(<120 \mathrm{~m})$, medium (between 120 and $200 \mathrm{~m})$ and large $(>200 \mathrm{~m})$. User intervened preference weights for vessel classes are given as 0.6; 0.3 and 0.1 for large, medium and small vessels, respectively. This was mentioned as a part of the port strategic policy reflecting agreements between shipping companies and the port. Nonetheless, port authorities still needed decision tools to guide them for meeting the requirements in the most efficient way. Policy D, previously explained in Sect. 3 is initially selected for the detailed explanation of the DSS implementation.

Next, vessel arrival data, vessel size, TEU loads, handling time of vessels should be entered into the system. When relevant information is put into the system, after the DSS has been run, the terminal manager will have supporting information as how to manage the allocation of berths to the incoming vessels. This information will be towards maximizing the throughput of the port. With regard to entering vessel related data, users have the alternatives of choosing between entering a fixed schedule considering the expected arrival times of vessels or a past data that will be used to calculate the appropriate distribution functions to be used for future arrivals.

To further clarify this input parameter, it should be mentioned that commonly, terminals operate with weekly Expected Time of Arrivals (ETA) provided by the shipping lines. This weekly data consisting of expected arrival date and time of each vessel together with TEU capacities may be supplied into the system through the user interface. These data are usually stored in common office programs such as MS Excel which can be given as an input into the proposed system. Alternatively, users may also pass past data related with vessel arrivals and container volumes.

The following experiments are based on past annual data between 01.01.2011 and 31.12.2011 including vessels' names, berthing times, departure times, vessel size, and TEU volume. By entering these data the system will calculate the appropriate distributions used within the model. It should be noted that ease of use has been one of the important requirements stated by terminal planners and accordingly the user does not need to provide the relevant distribution functions. Nevertheless, for detailed analysis, here, main distributions calculated by the system but kept optional for the users to check are as follows:

The best fit distribution for inter-arrival times in minutes is found as Gamma distribution with parameters $(349,1.17)$. Vessel size is normally distributed with mean 171 and standard deviation $35.2 \mathrm{~m}$. TEU amounts of vessels fits to the following Erlang distribution function: 0.999+ $\operatorname{ERLA}(288,2)$. TEU distributions for different vessel sizes classified into three categories and processing time functions based on past data with respect to TEU volumes calculated through regression analysis is shown in Table 2. The need for separating the processing times for each class has been specifically stated by the terminal managers due to the fact that different vessel classes may have specific restrictions which affects the processing times. For instance, number of cranes allocated to vessels is dependent on the vessel size and agreement between the shipping companies or larger vessels may require additional assistance and time while mooring.

As a next step, the decision variables, that is, the priority control indexes for vessel types should be calculated through the optimization module. A terminating condition should first be determined. For this, several pilot runs are conducted to give an indication of the performance of the optimization engine. Through trials, it has been observed that after 250 iterations very little improvement has been obtained. Taking a conservative approach, maximum number of iterations is set as 300 . The best solution obtained at the end of iterations determines the values of the decision variables. Using the optimization module, the priority control indexes for vessel types are found as 25, 23 and 20 for large, medium and small vessels respectively. 
Table 2 TEU amount and processing time distributions

\begin{tabular}{lll}
\hline Vessel class & TEU volume (in TEUs) & Processing times (h) \\
\hline Large vessels & $51+\operatorname{GAMM}(884+1,114)$ & $13.0+0.0161 * \mathrm{TEU}$ \\
Average vessels & $27+\operatorname{GAMM}(876+1,15)$ & $7.45+0.0267 * \mathrm{TEU}$ \\
Small vessels & $0.99+\operatorname{GAMM}(856+1,25)$ & $2.00+0.0683 * \mathrm{TEU}$
\end{tabular}

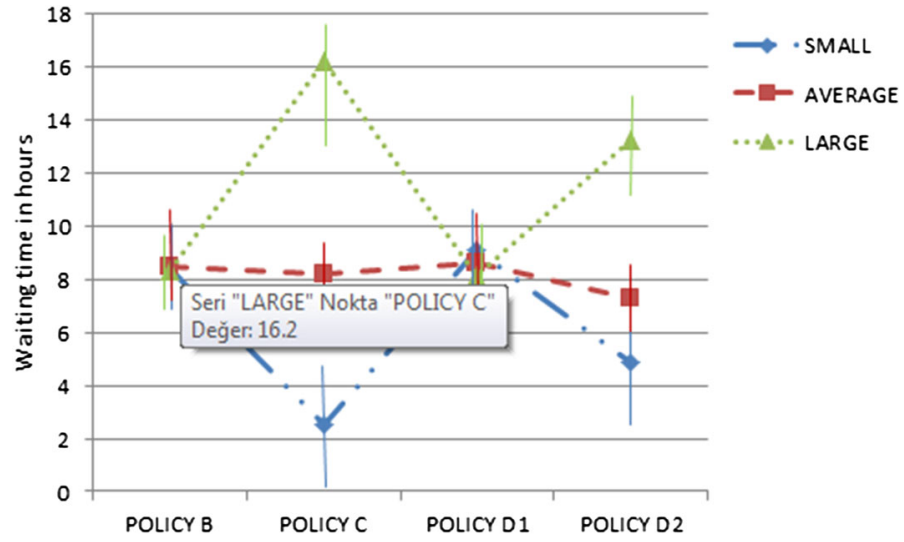

Fig. 7 Average waiting times under different berth allocation strategies

Further runs realized with 100 replications, setting replication length as 30 days, the throughput of the system is found as 106 vessels within a month. Average waiting times of vessels in queue for large, average and small ships are 9.1, 8.6 and 8.1 h. An important fact that caught the attention of the terminal planners was that by analyzing the relative priority control indexes for vessel classes although the port authority is in favour of larger vessels, it is also imperative to pay attention to the small vessels to increase the total throughput of the terminal.

To exemplify the potential use of the DSS in tactical decision making by the terminal planners, the effect of using different policies is demonstrated through further experiments. Figure 7 summarizes the model results of implementing different policies on waiting times for different vessel classes. Outcomes on waiting times for policies B, C and different configurations of Policy D reveal the effect of using different strategies. Policy B emphasizes the arrival times of vessels without considering vessel classes. Consequently, more balanced waiting time duration amongst vessel classes is observed. Policy $\mathrm{C}$ favours vessels of smaller classes giving priority to shortest processing times. Accordingly, shorter waiting times for smaller vessels are attained, whereas larger vessels are negatively affected by this decision policy. Policy D considers user intervened user preferences and also works on finding optimal threshold values for allocating vessels to the berths. The results reveal a balanced distribution of waiting times amongst vessel classes. To further analyze the effect of user intervened preferences, Policy D is examined under an additional setting, named D2, where user intervened preferences are omitted. From the results it can be observed that smaller vessels would benefit more from this strategy. The results comply with the previous study by Zhou and Kang (2006), where they stated that benefits are attainable in terminals where ship-to berth assignments are employed without the FCFS basis. The DSS developed in this study, details 
these benefits and provides the terminal planners an environment to analyze the outcome of different strategies. It is then up to the decision maker to implement a strategy that would be in line with the port strategy and support their agreements with shipping companies.

\subsubsection{Increased traffic volume}

It is also important to consider the use of the DSS in larger ports in the world, such as within the Port of Rotterdam. Further experiments are based on vessel traffic density between 01.01.2013 and 01.06.2013 for the Uniport Container Terminal within the Port of Rotterdam. This corresponds to approximately twice as many vessel arrivals compared to the container terminal in Izmir. The inter-arrivals are distributed with Gamma (173,1.15). Similar berth characteristics are assumed in order for the terminal planners to predict future state with increased traffic. The user intervened preference weights are given as equal for each of the vessel classes which implies that there is no user preference weight for different vessel classes.

In this setting, running the optimization module gives the values of priority increases for vessel classes as 95, 30 and 5 for small, medium and large vessels respectively. The average throughput of the system is increased to 213 vessels. Average waiting times are recorded as 8.47, 16.67 and $37.81 \mathrm{~h}$ for small, medium and large vessels respectively. One important difference as compared to the previous experiment is that with the absence of user intervened preference factors for each vessel class, relative priority control indexes show higher variations. According to the results, it can be seen that giving more preference to smaller vessels is more beneficial for maximizing the throughput of the system. However, it is still important to check the system's behaviour under each different terminal setting and parameter values.

\subsection{Expansion of the port facilities}

Subsequently, further experiments are conducted under different terminal settings. Expansion plans of the port of Izmir are considered. Total value of investments is calculated approximately as 170 million US dollars. An important aspect with respect to the berthing decisions with the expansion of the port is that due to the additional quays that will be constructed outside the peninsula formed space, berths are expected to have different handling times with different equipment used and dissimilar distances to the container storage yards. This leads to changes in the service time and parameters. This terminal type refers to terminals where there are clear differences among berths. In this setting a solution procedure where different handling times of vessels berthed at different quays should be considered. A vessel will receive faster service when berthed at faster quays. This type of setting is generally common among terminals around the world where a continuous quay space structure is present. The new structure should be handled correctly under the proposed DSS. Policy E, which considers berth classes, is adopted for the berth allocation strategy.

To further explore the capabilities of the DSS, in the following experiments, vessels are classified according to their TEU volumes. If a vessel is carrying less than or equal to 500 TEUs it will be referred to as small. Likewise, in case of a TEU volume of between 500 and 1000 TEUs it will be characterized as medium, and for larger volumes it will be called as a large vessel. User intervened preference weights for vessel classes are given as 0.6; 0.3 and 0.1 for large, medium and small vessels, respectively.

Within the future expansion plans the berths that are expected to have different handling rates that may be classified as fast, medium and slow. The processing times set by the support of terminal planners are given in Table 3. 
Table 3 Processing time for each vessel class

\begin{tabular}{llll}
\hline Vessel types & Fast berth & Medium berth & Slow berth \\
\hline Large & $15+0.0114 *$ TEU & $25.2+0.0114 *$ TEU & $35+0.0114 *$ TEU \\
Medium & $1+0.0372 *$ TEU & $2.06+0.0372 *$ TEU & $5+0.0372 *$ TEU \\
Small & $3+0.0292 *$ TEU & $6.15+0.0292 *$ TEU & $9+0.0292 *$ TEU \\
\hline
\end{tabular}

With the increased quay space, testing the model under current traffic of the Port of Izmir, leads to no significant waiting times of vessels. Nearly all vessels may be served without the occurrences of waiting lines. Due to this already anticipated result, further experiments are realized under the increased traffic volume given in the earlier experiments.

Basically, with the existence of berths with different classes, all vessels will try to enter the berths with the fastest processing times. However, in case of congestion, waiting lines will occur and priority control mechanism must be applied for berth planning. Each vessel class will have to wait at the port horizon until the determined threshold level for the vessel class has been met. This will be determined through the user intervened vessel class preferences. Subsequently, waiting lines of the queues will be managed by the use of the berth-class pair threshold values computed through the optimization model. Execution of the optimization algorithm provides the threshold values for berth-vessel class pairs, named v1 through v9 as $3,6,10,5,8,11,7,11,12$, respectively. These values correspond to number of vessels waiting in the queues. For instance a medium class vessel will get serviced at a fast berth if the number of vessels in the fast class queue is less than or equal to the threshold value of v4. It will be serviced at a medium berth if the number of vessels in the fast queue is larger than v4 and number of vessels in the medium queue is less than or equal to v5. Finally, it will be serviced at a slow berth if number of vessels in the fast queue is larger than $\mathrm{v} 4$ and number of vessels in the medium queue is larger than v5 and number of vessels in the slow queue is less than or equal to v6. The experiment results show that together with the preference of the large vessels the DSS balances the allocation of berths among vessel classes.

Using policy E, average service times including waiting and processing durations are found as approximately $39.5,33.54$ and $27.88 \mathrm{~h}$ for large, medium and small vessels. To evaluate the effect of berth allocation policy on these outcomes experiments are continued with adopting Policy $\mathrm{C}$ where differences among vessel and berth classes are not considered during allocation phase. Comparison data are shown in Table 4. Outcomes reveal that waiting times of vessels classes of large and medium are increased when Policy $\mathrm{C}$ is implemented. This is mainly due to the fact that Policy $\mathrm{C}$ favours small vessels without considering priority preferences among vessels. Smaller vessels are first released from the queue and allocated to the first available berth without considering berth rate differences. The results suggest that adopting different strategies will lead to changes in port performance figures. Developed DSS allows terminal managers to experiment different strategies and aid them through the decision of implementing the most suitable strategy.

\subsection{Experimentation summary}

The experiments are conducted on Intel I5 processor with $2.53 \mathrm{GHz}$ clock. The response time of the DSS varies between 2 and 7 min for the presented experiments. It should be noted that as simulation is incorporated in the evaluation phase of the scatter search procedure and several 
Table 4 Model outcomes for Policy $\mathrm{C}$ and $\mathrm{E}$

\begin{tabular}{llcll}
\hline Vessel types & \multicolumn{2}{l}{ Waiting time $(\mathrm{h})$} & \multicolumn{2}{l}{ Processing time $(\mathrm{h})$} \\
\cline { 2 - 5 } & Policy C & Policy E & Policy C & Policy E \\
\hline Large & 37.81 & 4.04 & 42.73 & 35.47 \\
Medium & 16.67 & 5.68 & 27.70 & 27.86 \\
Small & 8.46 & 15.14 & 13.82 & 12.75 \\
\hline
\end{tabular}

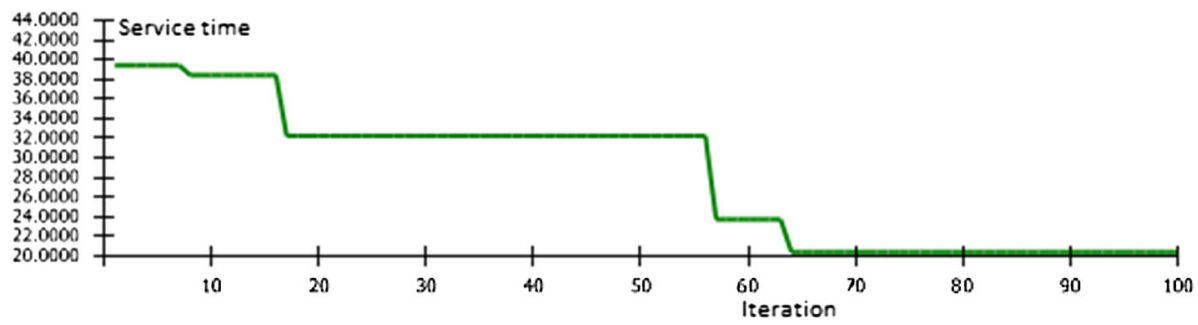

Fig. 8 Algorithm processing display

number of replications are required to obtain a reliable estimate of the objective function, the complexity of the simulation model plays an important role in the running time of the model. Higher running times are observed when for instance complex yard configurations are set. Figure 8, shows the user interface where the processing of the algorithm can be observed dynamically during the progress of the program. As an option, the user may interrupt the process and obtain the best results obtained so far in case immediate results are required. In the main, the running times are found to be reasonable and acceptable for practical use by the terminal operators of the port.

Experimentations show that possible reductions in vessel service times are possible through the use of berth allocation policies. Specific to the terminal configuration the results suggest more efficient use of berthing facilities and allow the users to control the customer service levels with respect to the shipping companies.

It should be noted that with the complexity of the system and the stochastic nature of the arrival and service rates it may be misleading to make straightforward assumptions on the behaviour of the system. Any change made in the preference weights, vessel arrivals or berth handling rates will certainly affect these results. Accordingly the DSS model results should be analyzed for each container terminal setting. Consequently, the aim of the experiments demonstrated here is to demonstrate the usage and show the capabilities of the DSS. Although we have realized extensive observations at the Port of Izmir the two different types of terminals studied here are generic for other ports in the world as well. Furthermore, analysing the model's use in higher traffic enables the observation of the capabilities of the DSS in larger ports around the world.

\section{Conclusion}

Today, importance of ports is increasing along with the increase of global trade volume. This situation makes the efficiency of port management under the capacity constraints a critical issue. Accordingly, berth allocation problem has become further significant due to 
being the root of all operational problems within a port. With its complexity, port authorities demand for effective decision support tools to support this decision making process. Due to this necessity, a DSS is designed for the BAP, in collaboration with the port authorities. The problem focused is the BAP considering vessel preferences and port objectives.

The literature is extended by developing a DSS that manages the priority control mechanism reflecting the practical considerations and needs. A new model is designed using simulation and optimization techniques. Embracing further facts linked with the berth allocation problem and service priorities adds to the body of knowledge making the model more convenient for real-world use. The DSS is designed to provide a high degree of flexibility with a good level of parameterization allowing users to work under different terminal scenarios and analyse situations in depth. Terminal planners can use the DSS for tactical and strategic level decisions. The effects of changes in the terminal infrastructure can be investigated through the use of the model.

The expansion of the DSS to include yard operations is an opportunity for a future work. A model that will consider priority factors for the storage space allocation of containers within the yard will enable the storage resources to be used more efficiently. In this scene, the priority control mechanism can be enhanced to consider the detailed analysis of container movements connected with the external gateways. This will enable the port authorities to obtain general improvements in performance figures. However, remarkably increased complexity will be a challenge to manage in this circumstance.

Acknowledgements The generous assistance and valuable information provided by the employees of Port of Izmir and especially by Metin Ozyllmaz who provided excellent information and support throughout the project are sincerely acknowledged.

Conflict of interest The authors decalre that they have no conlict of interest.

Open Access This article is distributed under the terms of the Creative Commons Attribution 4.0 International License (http://creativecommons.org/licenses/by/4.0/), which permits unrestricted use, distribution, and reproduction in any medium, provided you give appropriate credit to the original author(s) and the source, provide a link to the Creative Commons license, and indicate if changes were made.

\section{References}

Bandeira, D. L., Becker, J. L., \& Borenstein, D. (2009). A DSS for integrated distribution of empty and full containers. Decision Support Systems, 47(4), 383-397.

Boile, M., Theofanis, S., \& Golias, M. (2006, December). Berth allocation with service priorities: A linear reformulation. In Proceedings of the 5th WSEAS/IASME international conference on system science and simulation in engineering (ICOSSSE 2006) (pp. 16-18). Tenerife, Canary Islands, Spain.

Boile, M., Theofanis, S., Golias, M., \& Coitb, D. (2007). Berth planning by customer service differentiation: A multi-objective approach. In 11th World conference on transport research.

Canonaco, P., Legato, P., Mazza, R. M., \& Musmanno, R. (2008). A queuing network model for the management of berth crane operations. Computers \& Operations Research, 35, 2432-2446.

Carlo, H. J., Vis, I. F., \& Roodbergen, K. J. (2013). Seaside operations in container terminals: Literature overview, trends, and research directions. Flexible Services and Manufacturing Journal, 27(2-3), 224262.

Cordeau, J. F., Laporte, G., Legato, P., \& Moccia, L. (2005). Models and tabu search heuristics for the berthallocation problem. Transportation Science, 39(4), 526-538.

Du, Y., Xu, Y., Chen, Q. (2010). A feedback procedure for robust berth allocation with stochastic vessel delays, Center for Logistics Technology, Nankai University, Tianjin 300071, P. R. China. 
Glover, F., Kelly, J. P., \& Laguna, M. (1996). New advances and applications of combining simulation and optimization. In: J. M. Charnes, D. J. Morrice, D. T. Brunner, \& J. J. Swain (Eds.), Proceedings of the 1996 winter simulation conference (pp. 144-152).

Golias, M., Boile, M., \& Theofanis, S. (2006). The berth allocation problem: a formulation reflecting time window service deadlines. In Proceedings of the 48th transportation research forum annual meeting. Boston (MA): Transportation Research Forum.

Golias, M., Boile, M., \& Theofanis, S. (2007). The stochastic berth allocation problem. In Proceedings of the international conference on transport science and technology (TRANSTEC 2007) (pp. 52-66).

Guan, Y., \& Cheung, R. K. (2004). The berth allocation problem: Models and solution methods. OR Spectrum, 26(1), 75-92.

Guan, Y., Xiao, W. Q., Cheung, R. K., \& Li, C. L. (2002). A multiprocessor task scheduling model for berth allocation: Heuristic and worst-case analysis. Operations Research Letters, 30(5), 343-350.

Han, X. L., Lu, Z. Q., \& Xi, L. F. (2010). A proactive approach for simultaneous berth and quay crane scheduling problem with stochastic arrival and handling time. European Journal of Operational Research, 207(3), 1327-1340.

Hansen, P., Oğuz, C., \& Mladenović, N. (2008). Variable neighborhood search for minimum cost berth allocation. European Journal of Operational Research, 191(3), 636-649.

Imai, A., Chen, H. C., Nishimura, E., \& Papadimitriou, S. (2008). The simultaneous berth and quay crane allocation problem. Transportation Research Part E: Logistics and Transportation Review, 44(5), 900920 .

Imai, A., Nishimura, E., \& Papadimitriou, S. (2003). Berth allocation with service priority. Transportation Research Part B: Methodological, 37(5), 437-457.

Imai, A., Nishimura, E., \& Papadimitriou, S. (2008). Berthing ships at a multi-user container terminal with a limited quay capacity. Transportation Research Part E: Logistics and Transportation Review, 44(1), $136-151$.

Imai, A., Zhang, J. T., Nishimura, E., \& Papadimitriou, S. (2007). The berth allocation problem with service time and delay time objectives. Maritime Economics \& Logistics, 9(4), 269-290.

Kim, K. H., Lee, K. M., \& Hwang, H. (2003). Sequencing delivery and receiving operations for yard cranes in port container terminals. International Journal of Production Economics, 84(3), 283-292.

Kirpatrick, S., Gelatt, C. D., \& Vecchi, M. P. (1983). Optimization by simulated annealing. Science, 220, $671-681$.

Lai, K. K., \& Shih, K. (1992). A study of container berth allocation. Journal of Advanced Transportation, 26(1), 45-60.

Moorthy, R., \& Teo, C. P. (2006). Berth management in container terminal: The template design problem. OR Spectrum, 28, 495-518.

Murty, K. G., Liu, J., Wan, Y. W., \& Linn, R. (2005). A decision support system for operations in a container terminal. Decision Support Systems, 39(3), 309-332.

Ngai, E. W., Cheng, T. E., Au, S., \& Lai, K. H. (2007). Mobile commerce integrated with RFID technology in a container depot. Decision Support Systems, 43(1), 62-76.

Parola, F., \& Sciomachen, A. (2005). Intermodal container flows in a port system network: Analysis of possible growths via simulation models. International Journal of Production Economics, 97(1), 75-88.

Saharidis, G. K. D., Golias, M. M., Boile, M., Theofanis, S., \& Ierapetritou, M. G. (2010). The berth scheduling problem with customer differentiation: A new methodological approach based on hierarchical optimization. The International Journal of Advanced Manufacturing Technology, 46(1-4), 377-393.

Sargent, R. G. (2005, December). Verification and validation of simulation models. In Proceedings of the 37th conference on winter simulation (pp. 130-143). Winter Simulation Conference.

Shen, W. S., \& Khoong, C. M. (1995). A DSS for empty container distribution planning. Decision Support Systems, 15(1), 75-82.

Shi, J., Katehakis, M. N., Melamed, B., \& Xia, Y. (2014). Optimal continuous replenishment for inventory systems with compound poisson demands and lost-sales. Operations Research, 6(5), 1048-1063.

Stahlbock, R., \& Voß, S. (2008). Operations research at container terminals: A literature update. Or Spectrum, $30(1), 1-52$.

Steenken, D., Voß, S., \& Stahlbock, R. (2004). Container terminal operation and operations research-A classification and literature review. OR spectrum, 26(1), 3-49.

Vis, I. F. (2006). A comparative analysis of storage and retrieval equipment at a container terminal. International Journal of Production Economics, 103(2), 680-693.

Vis, I. F. A., \& De Koster, R. (2003). Transshipment of containers at a container terminal: An overview. European Journal of Operational Research., 147, 1-16.

Yun, W. Y., \& Choi, Y. S. (1999). A simulation model for container-terminal operation analysis using an object-oriented approach. International Journal of Production Economics, 59(1), 221-230. 
Zhen, L., Lee, L. H., \& Chew, E. P. (2011). A decision model for berth allocation under uncertainty. European Journal of Operation research, 212(2011), 54-68.

Zhou, P., \& Kang, H. (2008). Study on Berth and Quay-crane allocation under stochastic environments in container terminal. Online English Edition of The Chinese Language Journal SETP, 2008(28), 161-169.

Zhou, B., Katehakis, M. N., \& Zhao, Y. (2009). Managing stochastic inventory systems with free shipping option. European Journal of Operational Research, 196(1), 186-197.

Zhou, B., Zhao, Y., \& Katehakis, M. N. (2007). Effective control policies for stochastic inventory systems with a minimum order quantity and linear costs. International Journal of Production Economics, 106(2), 523-531. 